\title{
COST-EFFECTIVE SHARPENING OF SINGLE-SENSOR CAMERA IMAGES
}

\author{
Rastislav Lukac and Konstantinos N. Plataniotis \\ Multimedia Laboratory - BA 4157, The Edward S. Rogers Sr. Department of ECE, \\ University of Toronto, 10 King's College Road, Toronto, Canada \\ lukacr@ieee.org,kostas@dsp.utoronto.ca
}

\begin{abstract}
This paper presents a cost-effective image sharpening solution suitable for single-sensor digital cameras. The proposed solution enhances the structural content of the sensor image data captured using a Bayer color filter array (CFA). Subsequent demosaicking of the enhanced CFA image produces a visually-pleasing demosaicked image without the need for expensive sharpening in the RGB color domain. Simulation studies indicate that the proposed imaging pipeline, which employs an image sharpening step before the actual demosaicking module, yields excellent performance and outperforms the conventional pipeline (demosaicking followed by full-color image sharpening) in terms of both subjective and objective image quality measures.
\end{abstract}

\section{INTRODUCTION}

Single-sensor digital cameras use a color filter array (CFA) [1] placed on top of a monochrome image sensor [2], usually a charge-coupled device (CCD) or complementary metal oxide semiconductor (CMOS) sensor, to capture the visual scene. Since each sensor cell has its own spectrally selective filter, the acquired CFA data constitutes a mosaic-like gray-scale image [1]. This image is transformed to a full-color image by a demosaicking process which uses spectral interpolation to estimate the missing color components in an image array and restore the true color information [3],[4].

Since the CFA under-samples the color information originally present in the captured visual scene, demosaicked images often suffer from various visual artifacts such as aliasing and color shifts [1]. To reduce these effects, camera manufacturers place a blurring filter in the optical path [2]. However, the filter reduces both the sharpness and the resolution of the captured image. To compensate for the image blurring caused by the camera optics and the sensor and to enhance the highfrequency content (edges and fine details) of the image, digital cameras improve the visual quality of the demosaicked output using image sharpening or high-pass type filtering techniques [2]. As an alternative to the conventional pipeline used to sharp demosaicked images (Fig.1a), we propose here an approach $(F i g .1 b)$ which employs an image sharpening step be-

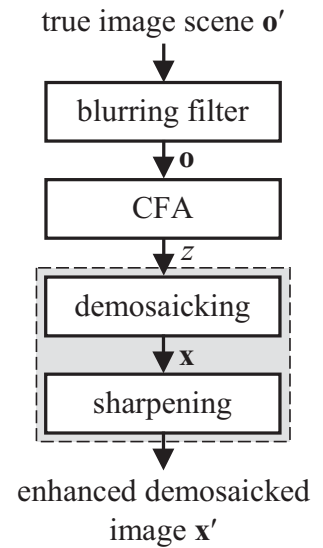

(a)

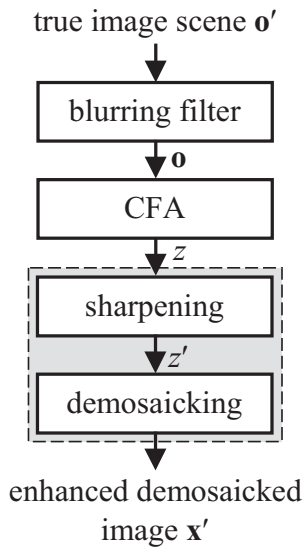

(b)
Fig. 1. Single-sensor imaging pipeline: (a) conventional approach, (b) proposed approach. Note that the input, intermediate and output images have the same resolution of $K_{1} \times K_{2}$ pixels.

fore the actual demosaicking module. This constitutes a costeffective processing solution capable of producing sharp demosaicked images. It will be shown that the proposed pipeline outperforms the traditional approach in terms of both performance and computational efficiency.

\section{SINGLE-SENSOR IMAGING BASICS}

The data acquired by the CFA-based sensor module constitutes a $K_{1} \times K_{2}$ gray-scale image $z: Z^{2} \rightarrow Z$ which has a mosaic-like structure (Fig.2a) due to CFA-driven acquisition of the visual scene. Thus, the spectral properties of pixels $z_{(r, s)}$ vary depending on the spatial location $(r, s)$, with the row coordinate $r=1,2, \ldots, K_{1}$ and the column coordinate $s=1,2, \ldots, K_{2}$. Using a widely accepted Bayer CFA with the GRGR phase in the first row (Fig.3) [1],[5], $z_{(r, s)}$ corresponds to the $\mathrm{R}$ component for (odd $r$, even $s$ ), the $\mathrm{G}$ component for (odd $r$, odd $s$ ) and (even $r$, even $s$ ), and the B component for (even $r$, odd $s$ ). As shown in Fig. $2 b$, demosaicking the CFA image $z$ produces a $K_{1} \times K_{2}$ Red-Green- 
(a)

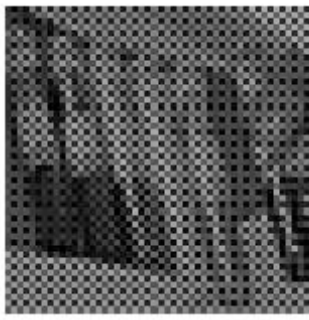

Fig. 2. Single-sensor imaging: (a) CFA image, (b) demosaicked full-color image.

Blue (RGB) color image $\mathrm{x}: Z^{2} \rightarrow Z^{3}$ with vectorial pixels $\mathbf{x}_{(r, s)}=\left[x_{(r, s) 1}, x_{(r, s) 2}, x_{(r, s) 3}\right]^{T}$. The value of the $\mathrm{R}$ $(k=1), \mathrm{G}(k=2)$, and $\mathrm{B}(k=3)$ component $x_{(r, s) k}$ denotes the contribution of the $k$-th primary in $\mathbf{x}_{(r, s)}$.

\section{CONVENTIONAL SHARPENING APPROACH}

To counteract the effect of low-pass type filtering operations performed during demosaicking, single-sensor digital cameras enhance color and tonal changes and edge transitions in the demosaicked image $\mathbf{x}$. Sharpening in-camera is based on fine enhancement operations to improve the perceived sharpness of captured images and avoid both the introduction of sharpening halos and the increase in the visibility of jaggedness, noise, and various demosaicking artifacts. Due to the real-time constraints, it is common to enhance $\mathbf{x}$ using the linear FIR filter as follows [2]:

$$
x_{(r, s) k}^{\prime}=x_{(r, s) k}+\sum_{(i, j) \in \zeta}\left\{w_{(i, j)} x_{(i, j) k}\right\}
$$

where $w_{(i, j)}$, for $(i, j) \in \zeta$, are coefficients of the high-pass filter operating in the area of support $\zeta$. For a common $3 \times 3$ window with $\zeta=\{(r-1, s-1),(r-1, s),(r-1, s+1),(r, s-$ $1),(r, s),(r, s+1),(r+1, s-1),(r+1, s),(r+1, s+1)\}$ the coefficients $w_{(i, j)}$ are given by

$$
\left[\begin{array}{ccc}
w_{(r-1, s-1)} & w_{(r-1, s)} & w_{(r-1, s+1)} \\
w_{(r, s-1)} & w_{(r, s)} & w_{(r, s+1)} \\
w_{(r+1, s-1)} & w_{(r+1, s)} & w_{(r+1, s+1)}
\end{array}\right]=\frac{1}{8}\left[\begin{array}{rrr}
-1 & -1 & -1 \\
-1 & 8 & -1 \\
-1 & -1 & -1
\end{array}\right]
$$

The signal outputted by $\sum_{(i, j) \in \zeta}\left\{w_{(i, j)} x_{(i, j) k}\right\}$ in (1) is a filtered high-frequency component of the local neighborhood $\zeta$ centered in $x_{(r, s) k}$. This component is added to the original signal $x_{(r, s) k}$ to increase the image sharpness and produce the enhanced signal $x_{(r, s) k}^{\prime}$. Based on the sliding filtering window concept [4], sharpening the whole image $\mathbf{x}$ requires that the procedure in (1) with (2) is repeated for $r=1,2, \ldots, K_{1}$, $s=1,2, \ldots, K_{2}$ and $k=1,2,3$, resulting in a $K_{1} \times K_{2}$ sharpened color image $\mathrm{x}^{\prime}: Z^{2} \rightarrow Z^{3}$ with the vectorial color pixels $\mathbf{x}_{(r, s)}^{\prime}=\left[x_{(r, s) 1}^{\prime}, x_{(r, s) 2}^{\prime}, x_{(r, s) 3}^{\prime}\right]^{T}$. By sharpening the demosaicked image, the framework depicted in Fig. Ia amplifies the visual impairments introduced by demosaicking.

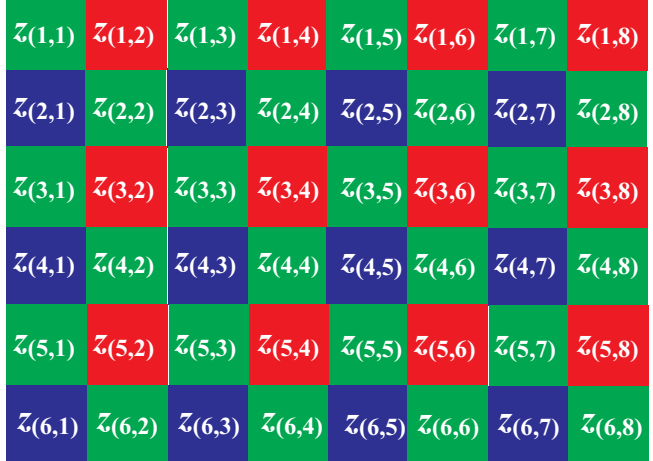

Fig. 3. Bayer CFA with the GRGR phase in the first row [5].

\section{PROPOSED SHARPENING APPROACH}

Unlike the conventional imaging pipeline (Fig.1a), we enhance directly the CFA image (Fig.1b). Thus, the proposed framework does not amplify the visual impairments introduced by demosaicking and generates visually pleasing, sharp, demosaicked images. To demonstrate the differences and ensure fair comparisons of the frameworks shown in Fig.1, the proposed here solution is dual to the sharpener defined in (1) and (2). Thus, we enhance the CFA image as follows:

$$
z_{(r, s)}^{\prime}=z_{(r, s)}+\sum_{(i, j) \in \zeta}\left\{w_{(i, j)} z_{(i, j)}\right\}
$$

where the enhanced data $z_{(r, s)}^{\prime}$ is constituted by the summation of the original CFA pixel value $z_{(r, s)}$ and the value of $\sum_{(i, j) \in \zeta}\left\{w_{(i, j)} z_{(i, j)}\right\}$ outputted by the high-pass filter operating over CFA samples $z_{(i, j)}$ in the neighborhood $\zeta$.

Since the CFA data constitutes the mosaic (Fig.2a), the shape mask $\zeta$ should follow the underlying CFA structure and restrict the area of support to the single locations $(i, j)$ which have the same color filters as the location under consideration $(r, s)$. This will ensure the desired enhancement of $z_{(r, s)}$. To overcome the problem, the CFA image sharpener in (3) is defined here using the shape mask $\zeta=\{(r-2, s-2),(r-$ $2, s),(r-2, s+2),(r, s-2),(r, s),(r, s+2),(r+2, s-$ $2),(r+2, s),(r+2, s+2)\}$ which implies the following coefficients:

$$
\left[\begin{array}{ccc}
w_{(r-2, s-2)} & w_{(r-2, s)} & w_{(r-2, s+2)} \\
w_{(r, s-2)} & w_{(r, s)} & w_{(r, s+2)} \\
w_{(r+2, s-2)} & w_{(r+2, s)} & w_{(r+2, s+2)}
\end{array}\right]=\frac{1}{8}\left[\begin{array}{ccc}
-1 & -1 & -1 \\
-1 & 8 & -1 \\
-1 & -1 & -1
\end{array}\right]
$$

Similarly to the Laplacian masks in (2), the mask in (4) possess the property that the sum of its elements is zero, to avoid intensity bias in the enhanced CFA image $z^{\prime}$. Based on (4), the value of $z_{(r, s)}^{\prime}$ in (3) is proportional to subtracting an average of neighboring CFA pixels from the central pixel $z_{(r, s)}$. This enhances an edges and fine details in high-frequency areas of the acquired CFA image, while outputting $z_{(r, s)}^{\prime}$ without a noticeable change compared to $z_{(r, s)}$ in smooth areas. 

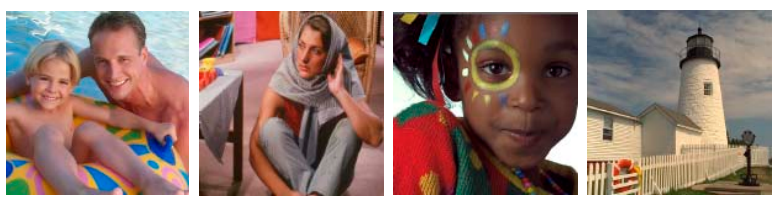

Fig. 4. Test color images Water, Barbara, Girl and Lighthouse.

By repeating (3) with (4) for $r=1,2, \ldots, K_{1}$ and $s=$ $1,2, \ldots, K_{2}$, the proposed solution generates a $K_{1} \times K_{2}$ enhanced CFA image $z^{\prime}: Z^{2} \rightarrow Z$ with gray-scale values $z_{(r, s)}^{\prime}$. Subsequent demosaicking of $z^{\prime}$ generates the enhanced demosaicked image $\mathbf{x}^{\prime}$. Since demosaicking is applied to the sharpened CFA image $z^{\prime}$, no additional sharpening in the demosaicked color domain is necessary. The proposed processing pipeline directly outputs the enhanced color image with visually sharp edges and fine details.

\section{EXPERIMENTAL RESULTS}

To determine the performance of camera image sharpeners, the test color images (Fig.4) with a $512 \times 512$ pixel resolution were blurred before sampling the visual information using the CFA. This simulates the real single-sensor imaging pipeline with the blurring/antialiasing filter [2]. Following the processing flow depicted in Fig.1, tests were performed by blurring the original images $\mathbf{o}^{\prime}$ with a $3 \times 3$ component-wise mean filter to produce the blurred color image $\mathbf{o}: Z^{2} \rightarrow Z^{3}$. The CFA image $z$ was obtained by sampling the image o with the Bayer CFA shown in Fig.3. The demosaicking solutions in [8]-[12] were employed in both processing pipelines to demosaick the CFA image $z$ (Fig.la) or its enhanced variant $z^{\prime}$ (Fig.1b). In the conventional pipeline (Fig.1a), an enhanced demosaicked image $\mathbf{x}^{\prime}$ was obtained from $z$ using demosaicking and color image sharpening steps. In the proposed pipeline (Fig.1b), $z$ was transformed to $\mathrm{x}^{\prime}$ using CFA image sharpening and demosaicking steps. Performance evaluations were comprised through comparisons of the original color image $\mathbf{o}^{\prime}$ to the enhanced demosaicked image $\mathbf{x}^{\prime}$. To facilitate the objective comparisons, the RGB color space based mean absolute error (MAE) [6] and mean square error (MSE) criteria [6], and the spatial color difference (SCD) criterion defined in the S-CIELab color space [7] were used.

Results reported in Tables 1 and 2 demonstrate that the proposed pipeline, based on CFA image sharpening, clearly outperforms the traditional solution which performs sharpening in the demosaicked RGB color domain. Since the significant improvement was observed along all employed demosaicking solutions and for all test images and objective measures, we can claim that the proposed approach is robust and suitable for single-sensor imaging devices. Among the demosaicking solutions considered in this paper, the solution presented in [12] produced the best results.

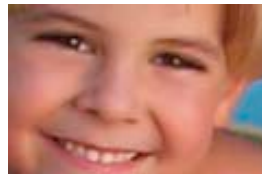

(a)

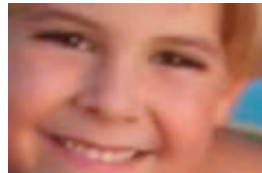

(b)

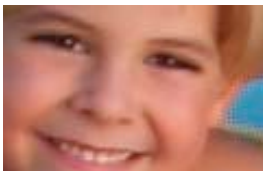

(c)

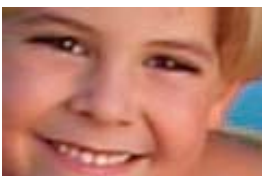

(d)

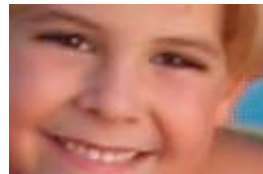

(e)

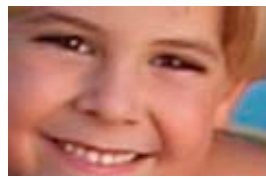

(f)
Fig. 5. Results corresponding to image Water: (a) original image $\mathbf{o}^{\prime}$, (b) blurred image $\mathbf{o}$, (c-f) restored image $\mathbf{x}^{\prime}$; (c) demosaicking in [8] followed by sharpening in (1), (d) sharpening in (3) followed by demosaicking in [8], (e) demosaicking in [12] followed by sharpening in (1), (f) sharpening in (3) followed by demosaicking in [12].

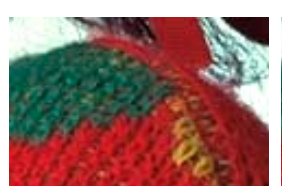

(a)

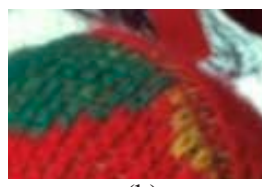

(b)

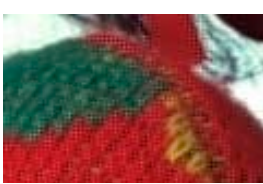

(c)

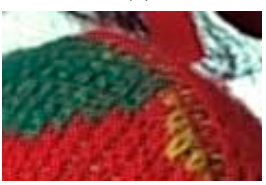

(d)

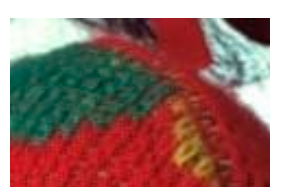

(e)

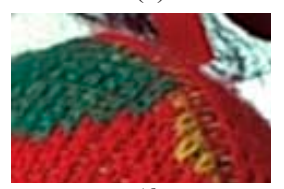

(f)
Fig. 6. Results corresponding to image Girl: (a) original image $\mathbf{o}^{\prime}$, (b) blurred image $\mathbf{o}$, (c-f) restored image $\mathbf{x}^{\prime}$; (c) demosaicking in [11] followed by sharpening in (1), (d) sharpening in (3) followed by demosaicking in [11], (e) demosaicking in [12] followed by sharpening in (1), (f) sharpening in (3) followed by demosaicking in [12].

Figs.5-7 depict enlarged parts of the achieved results. As it can be easily seen, the results produced by the traditional approach indicate the amplification of jaggedness, zipper effects and other demosaicking artifacts. On the other hand, the proposed approach produces visually pleasing images with higher visual quality and sharpness than the traditional approach. Our experimentation shows that the choice of the sharpening and demosaicking solutions and the order of their use during processing is essential for obtaining the highest visual quality and visually pleasing, sharp, camera images.

Apart from the actual performance, the proposed approach outperforms the traditional one also in terms of the computational efficiency. Since each channel of the demosaicked image represents a monochrome image alike the acquired CFA image, the use of the CFA image sharpening framework results in a three-fold reduction of the processing cost (the number of additions, multiplications, etc.) compared to the equivalent solution applied to the demosaicked image. When im- 
Table 1. Results corresponding to the traditional approach (demosaicking followed by color image sharpening).

\begin{tabular}{|c|ccc|ccc|ccc|cc|c|}
\hline Test image & \multicolumn{4}{|c|}{ Water } & \multicolumn{4}{|c|}{ Barbara } & \multicolumn{3}{c|}{ Girl } & \multicolumn{3}{c|}{ Lighthouse } \\
\hline Demosaicking & $M A E$ & $M S E$ & $S C D$ & $M A E$ & $M S E$ & $S C D$ & $M A E$ & $M S E$ & $S C D$ & MAE & MSE & $S C D$ \\
\hline method in [8] & 3.336 & 31.760 & 1.4382 & 8.480 & 193.597 & 2.7066 & 4.504 & 54.546 & 1.7149 & 6.591 & 140.462 & 2.1881 \\
method in [9] & 3.036 & 26.366 & 1.3499 & 8.324 & 188.456 & 2.6681 & 4.351 & 50.507 & 1.6833 & 6.571 & 138.520 & 2.1113 \\
method in [10] & 3.121 & 22.988 & 1.4262 & 8.476 & 192.529 & 2.7724 & 4.503 & 53.757 & 1.7390 & 6.624 & 139.485 & 2.1605 \\
method in [11] & 4.381 & 74.181 & 1.8660 & 8.849 & 203.461 & 2.8454 & 4.841 & 63.689 & 1.8497 & 6.748 & 143.516 & 2.1949 \\
method in [12] & 2.961 & 23.956 & 1.3044 & 8.281 & 188.088 & 2.5859 & 4.307 & 50.020 & 1.6240 & 6.481 & 137.098 & 2.0501 \\
\hline
\end{tabular}

Table 2. Results corresponding to the proposed approach (CFA image sharpening followed by demosaicking).

\begin{tabular}{|c|c|c|c|c|c|c|c|c|c|c|c|c|}
\hline Test image & \multicolumn{3}{|c|}{ Water } & \multicolumn{3}{|c|}{ Barbara } & \multicolumn{3}{|c|}{ Girl } & \multicolumn{3}{|c|}{ Lighthouse } \\
\hline Demosaicking & $M A E$ & $M S E$ & $S C D$ & $M A E$ & $M S E$ & $S C D$ & $M A E$ & $M S E$ & $S C D$ & $M A E$ & $M S E$ & $S C D$ \\
\hline method in [8] & 2.957 & 22.683 & 1.3107 & 7.866 & 165.948 & 2.4445 & 4.166 & 45.256 & 1.4079 & 6.195 & 121.202 & 2.1381 \\
\hline method in [9] & 2.628 & 17.848 & 1.2300 & 7.726 & 162.462 & 2.4560 & 4.062 & 42.735 & 1.4391 & 6.219 & 122.492 & 2.2713 \\
\hline method in [10] & 2.757 & 17.248 & 1.3135 & 7.831 & 164.478 & 2.5264 & 4.164 & 44.418 & 1.4767 & 6.241 & 121.351 & 2.2314 \\
\hline method in [11] & 3.370 & 37.359 & 1.5896 & 7.990 & 169.132 & 2.5124 & 4.282 & 48.173 & 1.5084 & 6.193 & 120.440 & 2.1445 \\
\hline method in [12] & 2.609 & 17.330 & 1.1783 & 7.696 & 161.687 & 2.3319 & 4.044 & 43.029 & 1.3837 & 6.133 & 120.287 & 2.1119 \\
\hline
\end{tabular}

plemented in software (MS Visual C++ 5.0), the execution of the developed programs, on an Intel Pentium IV $2.40 \mathrm{GHz}$ CPU, 512 MB RAM box with Windows XP operating system, took on average $0.391 \mathrm{~s}$ for the traditional solution and 0.180 $\mathrm{s}$ for the proposed solution to enhance a $512 \times 512$ image.

\section{CONCLUSION}

This paper presented an new single-sensor imaging pipeline which uses the sharpening step before the demosaicking module. By enhancing the high-frequency content of the acquired CFA image, the proposed framework avoids amplifying visual impairments introduced by demosaicking, produces demosaicked images with improved sharpness compared to the conventional approach and boosts the computational efficiency of the processing pipeline.

\section{REFERENCES}

[1] R. Lukac and K. N. Plataniotis, "Color filter arrays: Design and performance analysis," IEEE Trans. Consumer Electronics, vol. 51, pp. 1260-1267, November 2005.

[2] K. Parulski and K. E. Spaulding, "Color image processing for digital cameras," in Digital Color Imaging Handbook, (eds.) G. Sharma, CRC Press, Boca Raton, FL., pp. 728-757, 2002.

[3] B. K. Gunturk, J. Glotzbach, Y. Altunbasak, R. W. Schaffer, and R. M. Murserau, "Demosaicking: Color filter array interpolation," IEEE Signal Processing Magazine, vol. 22, pp. 44-54, January 2005.

[4] R. Lukac, B. Smolka, K. Martin, K.N. Plataniotis, and A.N. Venetsanopulos, "Vector filtering for color imaging," IEEE Signal Processing Magazine, vol. 22, pp. 74-86, January 2005.

[5] B.E. Bayer, "Color imaging array," U.S. Patent 3971 065, July 1976.

[6] R. Lukac, K. N. Plataniotis, D. Hatzinakos, "Color image zooming on the Bayer pattern," IEEE Trans. Circuit and Systems for Video Technology, vol. 15, pp. 1475-1492, Nov. 2005.
[7] B. Wandell, "S-CIELAB: A spatial extension of the CIE L*a*b* DeltaE color difference metric." [Online] http://white.stanford.edu/ brian/scielab/

[8] B. Gunturk, Y. Altunbasak, and R. Mersereau, "Color plane interpolation using alternating projections," IEEE Trans. Image Processing, vol. 11, pp. 997-1013, September 2002.

[9] S. C. Pei and I. K. Tam, "Effective color interpolation in CCD color filter arrays using signal correlation," IEEE Trans. Circuits and Systems Video Tech., vol. 13, pp. 503-513, June 2003.

[10] L. Chang and Y. P. Tang, "Effective use of spatial and spectral correlations for color filter array demosaicking," IEEE Trans. Consumer Electronics, vol. 50, pp. 355-365, May 2004.

[11] D. Alleyson, S. Susstrunk, and J. Herault, "Linear demosaicing inspired by the human visual system," IEEE Trans. Image Processing, vol. 14, pp. 439-449, April 2005.

[12] L. Zhang and X. Wu, "Color demosaicking via directional linear minimum mean square-error interpolation," IEEE Trans. Image Processing, vol. 14, pp. 2167-2178, December 2005.

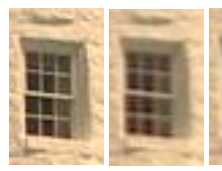

(a)

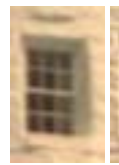

(b) (c)

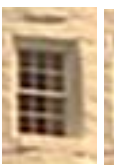

(d)

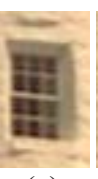

(e)

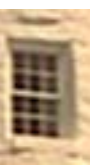

(f)

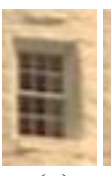

(g)

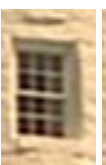

(h)

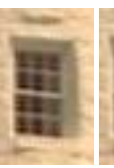

(i)

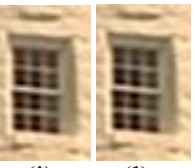

(j) (k)

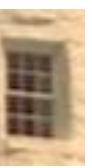

(1)
Fig. 7. Results corresponding to image Lighthouse: (a) original image $\mathbf{o}^{\prime}$, (b) blurred image $\mathbf{o},(\mathrm{c}-1)$ restored image $\mathbf{x}^{\prime}$; (c,e,g,i,k) demosaicked image sharpening, (d,f,h,j,l) CFA image sharpening; (c,d) demosaicking in [8], (e,f) demosaicking in [9], $(\mathrm{g}, \mathrm{h})$ demosaicking in [10], $(\mathrm{i}, \mathrm{j})$ demosaicking in [11], $(\mathrm{k}, 1)$ demosaicking in [12]. 